\title{
Diccionario histórico de terminología psicoanalítica
}

\section{Historical Dictionary of Psychoanalytic Terminology}

Mónica Vidal Díez [mvidaldiez@ub.edu]

Universitat de Barcelona, España

\begin{abstract}
RESUMEN
Aunque se ha progresado en muchos aspectos, aún faltan estudios sobre la historia del léxico especializado en español, sobre todo en la época moderna. Referente al lenguaje del psicoanálisis, existen, evidentemente, diccionarios de tipo enciclopédico a los que recurren los especialistas del ámbito, o repertorios léxicos que ofrecen la terminología con un enfoque puramente léxico. El objetivo principal de nuestra propuesta es avanzar en el ámbito del estudio del léxico de las lenguas de especialidad estudiando, por un lado, el léxico del psicoanálisis y, por el otro, elaborando un producto lexicográfico de carácter digital que permita la consulta de la información lingüística desde una perspectiva histórica. La propuesta se enmarca en un proyecto más amplio del grupo Neolcyt que lleva por título: "El léxico especializado del español contemporáneo". La metodología parte del estudio del léxico del psicoanálisis utilizado por Freud en sus obras, de su recepción, de su expansión, de su penetración en el idioma español, así como de su cotejo con lo ocurrido en otras lenguas.
\end{abstract}

\section{Palabras Clave}

Lexicografía; terminología; lenguas de especialidad; psicoanálisis, lexicografía funcional; lexicografía histórica

\footnotetext{
Abstract

Although progress has been made in many ways, there is still a lack of studies on the history of the specialized Spanish lexicon. Concerning the language of Psychoanalysis, there are encyclopedic dictionaries to which specialists in the field resort, or lexical repertoires that offer terminology with a purely lexical approach. The main objective of our proposal is to advance in the field of the study of the lexicon of Languages for Special Purposes by studying, on the one hand, the lexicon of Psychoanalysis and, on the other hand, developing a digital lexicographic product that allows the consultation of linguistic information from a historical perspective. The proposal is part of a larger Project of the Neolcyt group which is entitled: "El léxico especializado del español contemporáneo". The methodology starts from the study of the lexicon of Psychoanalysis used by Freud in his works, its reception, its expansion, its penetration into the Spanish language, as well as its comparison with what happened in other languages.
} 


\section{KEYWORDS}

Lexicography; terminology; languages for special purposes; psychoanalysis; function theory of lexicography; historical lexicography

RECIBIDO 2020-04-13; ACEPTADO 2020-08-21

Este proyecto se insiere en el Proyecto de Neolcyt colaborador con el Diccionario histórico de la Real Academia El léxico especializado en el español contemporáneo: 1884-1936 del Ministerio de Ciencia, Innovación y Universidades, PGC2018-093527-B-I00. Pueden consultarse los objetivos, los miembros que lo componen así como las publicaciones del grupo en la página web de Neolcyt en http://dfe.uab.es/neolcyt/. Asimismo, se presenta esta comunicación gracias a la ayuda del Grup de Lexicografia i Diacronia, grupo consolidado de investigación cuyo IP es la Dra. Glòria Claveria Nadal: 2017SGR-1251, http://www.lexicografia-diacronia. es/ca/index.htm.

Resulta paradójico que el país que cuenta hasta ahora con la mejor y más completa traducción de la obra de Freud, sea al mismo tiempo el país que muestra una mayor resistencia, más aún, una clara aversión a la implantación de la investigación psicoanalítica.

Antonio Abaúnza (1930: prólogo)

\section{Introducción}

Hasta hace poco, la filología tradicional se centraba casi de forma exclusiva en el estudio de la lengua literaria y de sus textos, sin atender apenas a las demás tipologías textuales. En los últimos años, sin embargo, ha ido creciendo el interés, entre otros, por los textos científico-técnicos como punto de partida para los estudios lingüísticos gracias a los trabajos de los historiadores de la ciencia quienes ponen de manifiesto la importancia de la divulgación científica en el ámbito social y su repercusión en la lengua general (Garriga 2009). Ya en 1972, Antonio Colino López (1972: 6), en su discurso de recepción a la Real Academia Española aseguraba que:

La irrupción de la Ciencia y de la Técnica ${ }^{1}$ en nuestra vida cotidiana ha creado un difícil problema en lo que se refiere al léxico vulgar o de general empleo. [...] El neologismo ya no se origina madurándose dentro de un ambiente científico, seguido quizás de una lenta penetración en el lenguaje vulgar, sino que surge en la noticia o en la información periodística y pronto se transforma en lenguaje común por la virtualidad de su uso.

En esta nueva aproximación al estudio de la lengua, los lingüistas comienzan a leer y a analizar los textos más importantes para la divulgación de cada ciencia, de cada disciplina, en cada época.

$1 \quad$ En mayúsculas en el original. 
Ahora bien, muchos de esos textos son precisamente traducciones (Garriga 2009). En esta situación se encuentra precisamente el lenguaje del psicoanálisis cuyos postulados parten de Sigmund Freud, el cual produjo toda su obra en alemán.

Pero el estudio de la lengua científica revela, asimismo, la historia social de la comunidad productora-receptora puesto que la actitud antes las 'palabras nuevas' - neologismos - descubre, también, la actitud ante las realidades por ellas representadas. Cualquier sistema organizado de representación de la naturaleza puede reflejar o explicar de qué manera interpreta la sociedad el orden y la experiencia social que la rodea. Siguiendo a Rubén (1993-1994: 39):

Un planteamiento multifuncional en el empleo del recurso explicativo de los intereses en el cambio científico contribuye a diluir los perjuicios de un demarcacionismo excesivamente rígido al mostrar cómo en el desarrollo científico se ponen en práctica tanto recursos técnicos predictivos y de control como recursos ideológicos legitimadores. En suma, la ciencia, como toda subcultura social, está afectada por los mismos elementos que cualquier otra subcultura social esotérica o especializada.

De ahí que el reflejo del léxico en los textos especializados y no especializados ponga de relieve, no solo la existencia de una nueva realidad científica patentizada a través del léxico, sino también la forma en cómo la sociedad recibe, acoge o rechaza esta nueva ciencia.

Como es sabido, el psicoanálisis ha sido creado y elaborado, por lo menos en sus postulados básicos por Sigmund Freud, un 'hombre como Dios' para sus admiradores, y un 'judío con complejo de inferioridad' para sus detractores. Si bien Freud es el punto de partida de esta nueva disciplina, la evolución del psicoanálisis era inevitable y necesaria entre otras muchas razones, porque Freud no podía utilizar en su investigación conocimientos de otras ciencias que en su época no existían. El proyecto que presentamos, sin embargo, se centra, por el momento de forma exclusiva, en sus postulados, esto es, se toma en consideración únicamente la terminología que el psicoanalista austríaco acuñó.

España es uno de los países europeos en los que menos influencia ha tenido el psicoanálisis y, paradójicamente, el castellano fue el primer idioma al que se tradujeron las obras completas de Sigmund Freud gracias a la labor conjunta de José Ortega y Gasset (1883-1955), introductor en España de todo lo que de interés ocurría en el mundo del pensamiento en alemán, José Ruiz-Castillo (1910-1945), presidente de la editorial Biblioteca Nueva, y Luis López-Ballesteros y de Torres (1896-1938), primer traductor al castellano de las obras completas de Freud. Pese a la resistencia que presentaron la Iglesia y algunos intelectuales de la primera mitad del siglo XX, los términos técnicos del psicoanálisis han ido penetrando poco a poco en nuestra lengua no solo como términos de especialidad, sino también como términos del uso común de los hablantes.

Existen, evidentemente, diccionarios de tipo enciclopédico de esta disciplina a los que recurren los especialistas del ámbito así como repertorios de tipo léxico. El proyecto que proponemos, no obstante, no corresponde a ninguna de ambas orientaciones; esto es, ni pretende abordar un enfoque enciclopédico ni, tampoco, una orientación meramente léxica. Nuestro propósito es confeccionar un proyecto lexicográfico que entronque directamente con las líneas de investigación del grupo Neolcyt. De ahí que la propuesta que planteamos se sitúe en tres ejes fundamentales: la lexicografía —elaboración de un diccionario digital—, la lengua especializada en el ámbito del psicoanálisis, y el estudio histórico del léxico (diacronía). 
Puesto que el proyecto se enmarca dentro del grupo Neolcyt en el desarrollo del Diccionario histórico del español moderno de la ciencia y de la técnica (DHEMCYT) ${ }^{2}$ y este, a su vez, colabora con el Diccionario histórico de la Real Academia en el ámbito de la ciencia y de la técnica, los resultados del estudio recubrirán en él, precisamente, una de las disciplinas: el psicoanálisis. Consecuentemente, los destinatarios target son los historiadores de la lengua y, en última instancia, los historiadores de la ciencia, sin descartar a quienes se dedican a la historiografía en lo que concierne a la historia de las ideas ${ }^{3}$.

\section{Objetivos}

El objetivo general que nos hemos propuesto es el de estudiar en profundidad el léxico del psicoanálisis - la terminología - utilizado por Freud en todas sus obras. Puesto que las obras originales del médico austríaco están redactadas en alemán, el acceso a los textos para el estudio del léxico en español pasa necesariamente por las traducciones que de las obras del psicoanalista se han realizado. Para ello, según se ha indicado, se partirá de las traducciones de López-Ballesteros así como de la traducción posterior que llevó a cabo Etxeberry para la editorial Amorrutu. Quizás es necesario recordar aquí, una vez más, el extraordinario trabajo realizado por López-Ballesteros no solo por la cualidad de sus traducciones sino, también, por el momento en que las llevó a cabo.

Desglosamos este en dos objetivos específicos:

1. El primero de ellos se propone analizar y describir la lengua especializada en el ámbito del psicoanálisis tomando como punto de partida la terminología que acunó Freud y su penetración en la lengua estándar hasta incorporarse al caudal léxico de los hablantes e introducirse en las obras lexicográficas.

2. El segundo objetivo persigue llenar el vacío existente en el ámbito del estudio del léxico de las lenguas de especialidad estudiando, por un lado, el léxico del psicoanálisis y, por el otro, elaborando un producto lexicográfico de carácter digital que permita la consulta de la información lingüística de los términos organizada en diferentes niveles de complejidad, en función de las necesidades de los usuarios. Recordamos, una vez más, que los usuarios target de nuestro proyecto son los estudiosos de la historia del léxico así como los historiadores de la ciencia.

Un trabajo como el que proponemos contribuirá a un mejor conocimiento de la historia reciente de las ideas lingüísticas en España acerca de los neologismos especializados, de la historia de esta ciencia en la Península, y aportará datos sobre las relaciones del español con las demás lenguas europeas.

$2 \mathrm{http} / / / \mathrm{dfe} . u a b . e s /$ neolcyt/index.php?option=com_flexicontent\&view=category\&cid=68\&Itemid=100\&lang=es

3 No especificamos la rama de esta historiografía por cuanto la historia de las ideas puede abordarse desde distintas disciplinas: filosofía, sociología, historia de la religión, historia de la medicina, etc. 


\section{Metodología}

Primeramente será necesario seleccionar los textos y proceder a un vaciado de voces e incorporarlas a un banco de datos léxicos ${ }^{4}$. Estos términos son los que más tarde constituirán el lemario de nuestro proyecto lexicográfico. Puesto que la terminología de esta ciencia ha surgido en alemán, será necesario acudir a las primeras traducciones que de las obras de Freud se han hecho en español. Asimismo, será oportuno contextualizar las voces tanto en alemán como en español, y llevar a cabo un estudio comparativo de cómo las palabras se han ido incorporando a los distintos idiomas. El cotejo se llevará a cabo sometiendo las voces a un estudio lexicológico que pasa por el rastreo de las voces en las obras lexicográficas de referencia de las lenguas alemana, española, inglesa, francesa e italiana. Como es sabido, las obras lexicográficas tienen un gran poder normativo y su estudio puede ayudar a la descripción de la lengua especializada. Por ello acudiremos, también, al NTLLE a fin de indagar la inclusión de los términos en la lexicografía tanto académica como no académica 5 . Por último, con el objetivo de comprobar cómo se ha divulgado la terminología de esta especialidad, estudiaremos la penetración de las voces en la prensa periódica, para lo cual acudiremos a la Hemeroteca Digital (HD) de la Biblioteca Nacional de España ${ }^{6}$, en línea, así como a la Biblioteca Virtual de la Prensa Histórica (BVPH), también en línea7.

Estas etapas de la metodología no son, sin embargo, lineales ni necesariamente sucesivas. En un primer momento, el acercamiento debe producirse con el propósito de establecer cuáles son las fuentes que se van a estudiar, qué diccionarios se van a tomar como referencia, etc. En lo que concierne a los aspectos teóricos nos basamos en los postulados de la lexicografía funcional.

Con respecto al equipo de trabajo, contamos con la inestimable ayuda de los miembros del grupo Neolcyt y, más adelante, no se descarta la colaboración con otras universidades.

\section{Fases de desarrollo del diccionario y plan de trabajo}

Las fases de trabajo diseñadas para la elaboración del proyecto lexicográfico se han agrupado como sigue: fase lingüística, fase histórica, fase enciclopédica y, por último, la fase digital. Cada una de ellas se compone de las siguientes subtareas:

\section{Fase lingüística}

Selección de las obras

Vaciado de las obras y elección de los términos.

Confección del lemario

Selección de los contextos.

$4 \quad$ Véase el apartado 5 de nuestro trabajo.

5 En una fase más avanzada sería interesante llevar a cabo un estudio similar a lo ocurrido en las demás lenguas, esto es, en qué medida las voces van siendo sancionadas por las obras lexicográficas de referencia.

6 Los corpus léxicos disponibles son insuficientes a la hora de estudiar el léxico especializado ya que está escasamente representado en ellos. Por otra parte, en lo que concierne a las lenguas de especialidad, el rendimiento de los corpus generales es pobre dada la alta redundancia de los elementos de la lengua común en los propios textos no literarios. Contrariamente, los recursos digitales en la red, fundamentalmente en forma de prensa periódica digitalizada, son una herramienta de primer orden para el conocimiento del léxico. 


\section{Fase histórica}

Documentar la primera traducción de las voces en español. Aquí acudiremos a la primera traducción de las obras de Freud realizada por López-Ballesteros para la Editorial Biblioteca Nueva (1924), así como la de Etxeverry en Amorrutu editores (1967). El motivo por el cual nos basamos en ambas se debe a que López Ballesteros no llegó a traducir en su totalidad las obras de Sigmund Freud. La primera traducción completa es la Etxeverry de la editorial Amorrutu. Sin embargo, en el ámbito del psicoanálisis, esta segunda no está especialmente bien considerada. Aun así, puesto que la primera de ellas no se ha reeditado, es la que resulta más accesible ${ }^{8}$.

Comprobar las primeras inclusiones de la terminología en los repertorios lexicográficos de la lengua española, determinar la primera aparición de los términos en los diferentes idiomas y, por último,

Constatar la penetración en la prensa española.

\section{Fase enciclopédica}

Definición enciclopédica del término, escuelas que utilizan el término, relaciones con otros conceptos, bibliografía sobre el término, etc. En nuestro proyecto, solo la definición enciclopédica es de presencia obligatoria.

\section{Fase digital}

Desarrollo digital del proyecto lexicográfico: diseño de la herramienta informática y de la interfaz.

Las fases no son necesariamente sucesivas; así, por ejemplo, una vez vaciadas las obras y creado el lemario será interesante poder acceder al desarrollo digital para poder verter directamente la información en la herramienta. El plan de trabajo, lógicamente, se ciñe a las fases aquí esbozadas.

\section{Diseño de la ficha lexicográfica a la luz de la Teoría funcional de la lexicografía}

El diccionario que proyectamos está basado en el enfoque de la lexicografía funcional planteado por Sven Tarp (2015: 5):

Una función lexicográfica puede definirse como la asistencia que presta una obra lexicográfica para satisfacer los tipos específicos de necesidades de información puntual que pueda tener un tipo específico de posible usuario en un tipo específico de situación extra-lexicográfica. La asistencia a que se refiere se logra por medio de los datos lexicográficos detenidamente preparados y hechos accesibles para su consulta.

8 Con respecto a la traducción de la editorial Amorrutu, la mayoría de los profesionales del psicoanálisis o bien consultan el original, o bien prefieren las traducciones del inglés. En nuestra opinión, la traducción de López Ballesteros es de una gran calidad. 
La teoría funcional distingue entre tres tipos fundamentales de situaciones sociales relevantes para la lexicografía, lo que deriva en tres funciones diferentes, a saber: las comunicativas, las cognitivas y las operacionales (Tarp 2008). Las primeras son las más estudiadas e incluyen la producción, recepción, traducción, revisión y corrección de textos, tanto en la lengua materna como en una lengua extranjera. Estas son la que habitualmente aparecen en los diccionarios generales monolingües o bilingües.

Las situaciones cognitivas se refieren a situaciones donde una persona, por una u otra razón, necesita aumentar sus conocimientos sobre cualquier cosa, por ejemplo sobre la cultura general, la misma lengua o una disciplina especializada como el psicoanálisis. En general, los usuarios que acuden a un diccionario para aumentar sus conocimientos sobre algún tópico necesitan, sobre todo, definiciones en el sentido más amplio de la palabra de las cuales pueden extraer informaciones correctas y actualizadas. Es decir, como regla general, precisan de algo más que las breves definiciones terminológicas que muy a menudo facilitan los diccionarios (Fuertes-Olivera y Tarp 2008).

El tercer y último tipo de situaciones sociales donde pueden asistir los productos lexicográficos son las operacionales, es decir, situaciones en las que los futuros usuarios precisan de instrucciones para realizar algún tipo de operación física o intelectual, por ejemplo, para manejar una máquina o formular las cuentas anuales.

Teniendo en cuenta que el objetivo del proyecto lexicográfico es un análisis de la terminología psicoanalítica, de su recepción y expansión, y de su cotejo con lo ocurrido en otras lenguas, a la hora de confeccionar la ficha lexicográfica de los lemas tendremos en cuenta la función comunicativa y la función cognitiva, exclusivamente.

La ficha lexicográfica se ha diseñado de acuerdo con las funciones antes esbozadas por lo que recoge la siguiente información: el lema, la información comunicativa y la información cognitiva siguiendo el siguiente esquema:
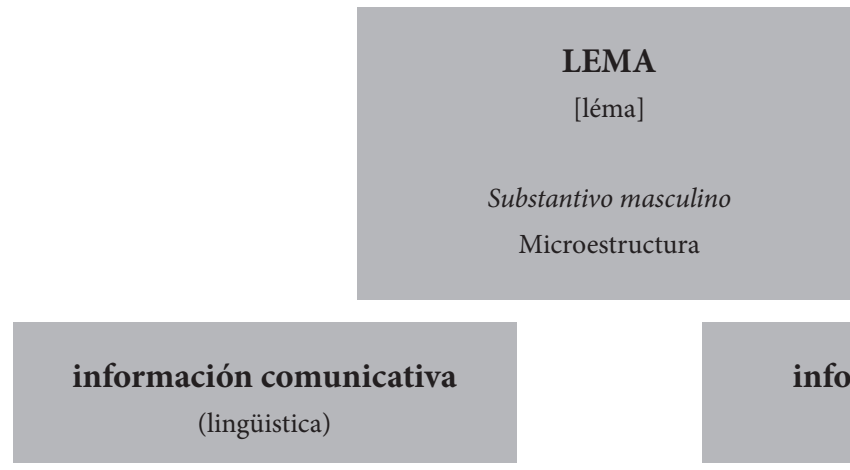

información cognitiva (especializada)

Esquema 1. Ficha lexicográfica. 
Cada uno de estos apartados contiene las siguientes especificaciones:

Lema $^{9}$

Categoría gramatical

Variantes gráficas

Datos comunicativos

Definición lexicográfica

Lema en la lengua original

Equivalencia en otras lenguas

Sinónimos

Variantes

Notas lexicológicas

Datos cognitivos

Contexto original

Información histórica: fecha de incorporación

Definición especializada

Notas enciclopédicas

En lo que concierne a los datos cognitivos y siguiendo los principios de la lexicografía funcional no se descarta el concurso del psicoanalista ${ }^{10}$.

\section{Herramienta informática: base de datos e interfaz ${ }^{11}$}

Para la confección de la base de datos (BD) nos basamos en la metodología que emplea Neolcyt para el proyecto en curso, adaptada al nuestro. Se trata de verter inicialmente toda la información en una hoja Excel, para luego subirla a una base de datos desarrollada con el sistema de gestión MySQL, cuyo lenguaje de programación usado para operar sobre dicha base de datos es el SQL (Structured Query Language), lenguaje estándar que sirve para administrar, consultar y elaborar los datos almacenados en una BD.

La primera cuestión será determinar qué campos van a figurar en cada una de las columnas de la hoja. Para ello partimos de los datos apuntados en la ficha lexicográfica, de acuerdo con cada una de las especificaciones. Alguna de ellas, por ejemplo, la equivalencia en otras lenguas, o los contextos en la $H D$ o en la $B V P H$, requerirá una subdivisión adicional atendiendo al número de idiomas propuesto o al número de fragmentos seleccionados (4). Dada la extensión de la plantilla,

9 Puede añadirse en este apartado la transcripción fonética y una pista de audio, esto último teniendo en cuenta que se trata de un diccionario digital.

10 La autora se ha valido de una psicoanalista para determinar algunas cuestiones, sobre todo en lo que concierne al análisis de los contextos de la prensa digital.

11 Para el desarrollo de este apartado quiero agradecer la impagable ayuda y colaboración de mi compañero de proyecto, el Dr. Pedote. 
en el esquema que se ofrece, algunos campos se sugieren con etc. siguiendo el esquema previamente marcado. El vertido en la plantilla de la base de datos queda como sigue ${ }^{12}$ :

\begin{tabular}{|c|c|c|c|c|c|}
\hline id & lema & marca_tecnica & categoria_gramatical & variante & sinónimo \\
\hline
\end{tabular}

cita bibl. original

fecha de acuñación term.

cita bibl. $1^{\text {a }}$ trad. cast.

fecha $2^{\mathrm{a}}$ trad. cast.

contexto $2^{\mathrm{a}}$ trad. cast. fecha ler. registro lex. esp.

$1^{\circ}$ doc. $H D$ fecha $1^{\text {a }}$ trad. fr.

contexto $1^{\text {a }}$ trad. fr. etc. cita bibl.1 ${ }^{\text {a }}$ doc. HD etc.

$1^{\text {a }}$ doc. $B V P H$

\section{\begin{tabular}{l|l|l|l} 
Def. espec. I & Fuente def. esp. I & Def. espec. II & Fuente def. esp. II $\quad$ Notas encicl.
\end{tabular}}

Esquema 2. Base de datos.

A fin de facilitar el trabajo a los miembros del equipo, se procederá a la división de la plantilla por áreas de interés, idioma, o especificación, según convenga y según la idiosincrásica de los miembros. Al final se ensamblará toda la información en una única tabla, base de datos, para someterla a su elaboración informática y confeccionar la interfaz. Esta deberá permitir la consulta indiscriminada de acuerdo con los posibles intereses del usuario partiendo del diseño que hemos propuesto. Esto último requiere el concurso de un (equipo) informático.

\section{Ejemplo}

Para ilustrar los contenidos de la rúbrica léxica y de acuerdo con la ficha lexicográfica diseñada, pasamos seguidamente a ejemplificar la voz Superyó.

Lema

Superyó

Categoría gramatical: sust. masc.

12 Se disponen los campos de esta forma por ser imposible su presentación en un único renglón ya que en total se trata de 33 columnas más los campos elididos. Por idénticas razones, tampoco es posible reflejar los contenidos de la base de datos tal y como aparece en la hoja Excel. 
Variantes gráficas: súper yo, superyó, super-yo, super-Yo.

[Transcripción fonética [su.per.' jo] y pista audio, ambas opcionales]

Datos comunicativos

Definición lexicográfica: m. Psicol. En el psicoanálisis freudiano, parte inconsciente del yo que se observa, critica y trata de imponerse a sí mismo por referencia a las demandas de un yo ideal. Lema en la lengua original: Über-ich

Equivalencia en otras lenguas: Al: Über-Ich. - Fr.: surmoi (o sur-moi). —Ing.: super-ego. —II.: super-io. - Por.: superego, - Cat.: superió.

Sinónimos: yo ideal,

Variantes: superego

Notas lexicológicas: en la definición del término, la Rae remite directamente al término superego. El concepto de Yo ideal (en alemán, Ichideal) figura ya en la obra de referencia de Freud.

Datos cognitivos

Contexto original en alemán: Siegmund Freud (1923): Leipzig, Wien u. Zurich: Internationaler Psychoanalytischer Verlag.

Das Ich und das Es. Kapitel 3.

III. Das Ich und das Über-Ich (Ichideal)

Wäre das Ich nur der durch den Einfluß des Wahrnehmungssystems modifizierte Anteil des Es, der Vertreter der realen Außenwelt im Seelischen, so hätten wir es mit einem einfachen Sachverhalt zu tun. Allein es kommt etwas anderes hinzu. Die Motive, die uns bewogen haben, eine Stufe im Ich anzunehmen, eine Differenzierung innerhalb des Ichs, die Ich-Ideal oder Über-Ich zu nennen ist, sind an anderen Orten auseinandergesetzt worden zur Einführung des Narzißmus, Massenpsychologie und Ich-Analyse.

\section{Información histórica:}

Primera traducción al castellano: (1924) El yo y el ello, Traducción de Luis López-Ballesteros, (17 vols.), 9, 237-96, BN.

\section{Contexto:}

El yo y el super-yo (ideal del yo)

Si el Yo no fuera sino una parte de Ello modificada por la influencia del sistema de las percepciones, o sea, el representante del mundo exterior, real, en lo anímico, nos encontraríamos ante un estado de cosas harto sencillo. Pero hay aun algo más. Los motivos que nos han llevado a suponer la existencia de una fase especial del Yo, o sea, de una diferenciación dentro del mismo Yo, a la que damos el nombre de Super-Yo o ideal del Yo, han quedado ya expuestos en otros lugares.

Segunda traducción al castellano: José Luis Etxeverry, Obras completas, Ediciones Amorrutu, Buenos Aires, (1976) 


\section{Contexto:}

El yo y el superyó (ideal del yo)

Si el yo fuera solo la parte del ello modificada por el influjo del sistema percepción (sic), el subrogado del mundo exterior real en lo anímico, estaríamos frente a un estado de cosas simple. Pero se agrega algo más. En otros textos se expusieron los motivos que nos movieron a suponer la existencia de un grado (Stufe, también 'estadio') en el interior del yo, una diferenciación dentro de él, que ha de llamarse ideal-yo o superyó.

Primera aparición en la lexicografía española: 1984 Academia usual

Primer registro en la literatura alemana: 1923

Contexto: (véase contexto original en alemán)

Primer registro en la literatura francesa: [TLF] (A. HESNARD, 1923, Les Récents enseignements psychol. de Freud, in L'Encéphale, sept.-oct., pp. 528-529 ds QUEM. DDL t. 29).

\section{Contexto:}

[...] [p. 528] laisser dans le Moi un dépôt, consistant en l'établissement de ces deux identifications en quelque sorte conciliées entre elles, et qui constitue un Moi idéal ou Sur-moi, opposé au reste de la personnalité, $[\ldots]$.

Primer registro en la literatura inglesa:

[OED super-ego] 1924. etc. [see $\mathrm{ID}^{2}$ ]. Also superego. "That part of the psyche wich controls the impulses of the id], as a translation of German Über-ich.

\section{Contexto:}

1938 Times Lit. Suppl, 26 Feb.132/4. «When the moral superego takes charge and the ego is no longer coercive but submissive».

Primer registro en la literatura italiana: [Cortelazzo e Zolli Super-Io] E. Morselli, La Psicanalisi: Studi ed appunti critici, Torino, I. 60. (1926) $)^{13}$

\section{Contexto:}

(E. Morselli 1926: 60) «Il Freud riconosce questa importanza direttiva della Coscienza vigile, evoluta, ad ora ne fa un altro Io, un 'Super-Io' o 'Io ideale'».

\section{Primeras documentaciones $H D$}

África. Revista de Tropas coloniales, 21 de junio de 1928. Gil Benumeya

Estas tres partes corresponden en lo esencial a la división de la ciencia psicoanalítica del hombre interior en ello (Cuerpo) yo (Espíritu) superyó (Alma). Terminaré esta digresión preliminar

13 Al parecer, el psicoanálisis no encontró un clima propicio en Italia. El propio Enrico Morselli (1852-1929), presidente de la Sociedad Italiana de Psiquiatría, había propiciado este clima de oposición al psicoanálisis publicando, casi tres lustros antes de la Segunda Guerra Mundial, un compendio en dos volúmenes — La Psicanalisi (sic.) (1926) en el que Freud era presentado como un "Satán lujurioso" y sus tesis sobre la sexualidad como un "catálogo pornográfico". De hecho, la obra de Morselli no es propiamente una traducción de las obras de Freud. 
excesivamente abstracta pero indispensable con la definición de los actos humanos (preliminar para comprender el llamado fatalismo musulmán estrechamente enlazado con la Baratía [...].

La Libertad, martes 5 de mayo de 1931, página 1. Luis Jiménez de Asua.

Defensa del 'vampiro' de Düsseldorf.

Son actos condicionados, en primera línea, por motivos inconscientes, respecto a los cuales la parte consciente do la personalidad no juega papel alguno. En la ejecución del hecho, el «yo» es dominado por mecanismos neuróticos especiales que debilitan la dependencia del «superyo ${ }^{14}$ ». Lo decisivo radica aquí en la existencia de esos mecanismos neuróticos particulares [...].

El Sol. 27 de octubre de 1932, página 2.

Instituto Español Criminológico.

Mañana, viernes, a las seis de la tarde, tendrá lugar en el Museo Antropológico (paseo de Atocha, núm. 13) la segunda lección del Curso de Psiquiatría Forense, que anualmente explica el doctor César Juarros. Tema: "El superyó y la autoexpiación”.

Luz. 24 de julio de 1933, página 8.

Deportes. Fútbol. Terminó la Asamblea.

Luis Otero. Cuando ya no es más que un concienzudo "barman", los prohombres del fútbol se acuerdan de él y le conceden la medalla del mérito. Con ella podrá epatar a sus clientes y perfeccionarse como superyó ante sus hijos, si los tiene.

Primeras documentaciones $B V P H$

La Libertad: La Libertad - Año XIII Número 3470 - 1931 mayo 5 (05/05/1931), pág. 1

Defensa del "vampiro" de Düsseldorf

En la ejecución del hecho, el «yo» es dominado por mecanismos neuróticos especiales que debilitan la dependencia del «superyo». Lo decisivo radica aquí en la existencia de esos mecanismos neuróticos $[\ldots]$

Hoja Oficial del lunes: editada por la Asociación de la Prensa - Año V Número 179 - 1934 abril 30 (30/04/1934), pág. 3

Los conflictos espirituales, causa de anormalidad infantil.

Expuso luego los conceptos "Comploxo de Edipo" (sic.) y "Super-Yo" de modo fácil de entender, destacando con textos de Moore, la importancia que en la aparición de las anomalías de conducta de los niños tiene la torpeza de la madre.

La Libertad: La Libertad - Año XVII Número 4858 - 1935 octubre 26 (26/10/1935), pág. 7

Dice que al surgir las dictaduras, con sus injusticias y atropellos, los individuos sienten relajarse la inhibitoria del super-yo, y que los que piden la pena de muerte demuestran tener un super-yo débil, un super-yo criminal potente, y constituyendo un acto de sadismo propio al que experimentan ciertas gentes al ver cómo es ajusticiado un reo.

14 Superyo, sin acento, en el original. 
El Adelanto: Diario político de Salamanca: Año 56 Número 17115 - 1940 enero 25, pág. 3

Conferencia de hoy en los Luises, por el catedrático Sr. Sánchez Tejerina.

[...] Versará sobre tema: "La Psicología (sic.) realista de los partidarios del psicoalálisis (sic.). El

"Yo", y el "Superyó" y el "Ello" en la determinación de los actos humanos, en especial del crimen.

Participación del "Ello" en el acto criminal. El "Superyó" criminal. El soborno de la conciencia. Una caprichosa interpretación de los sueños. La doctrina de las perversiones en los psicoanalistas y los endocrinólogos. [...] Salamanca, 25 de Enero de 1940.

Definición especializada:

Ofrecemos dos definiciones especializadas del término:

En el desarrollo de la personalidad, finalmente, se constituye una tercera instancia al separarse una parte del Yo y observarse a sí mismo, juzgando y criticando. Ya Freud del análisis de la manía de observación había deducido que en el ser existe una instancia que compara el actual Yo y cada una de sus manifestaciones, con un Yo ideal, forjado por él mismo en el curso de la vida debido, fundamentalmente, a la influencia ejercida por los padres y, secundariamente, por los maestros, medio ambiente, personas de significación para el sujeto, etc. A esta parte o función del Yo la llamó 'Súper Yo.' (Mandolini, 1969).

Laplanche-Pontalis (1996) introducen el concepto del siguiente modo, si bien el desarrollo del concepto en este diccionario es mucho más extenso:

Una de las instancias de la personalidad, descrita por Freud en su segunda teoría del aparato psíquico: su función es comparable a la de un juez o censor con respecto al yo. Freud considera la conciencia moral, la autoobservación, la formación de ideales, como funciones del superyó. Clásicamente el superyó se define como el heredero del complejo de Edipo; se forma por interiorización de las exigencias y prohibiciones parentales. Algunos psicoanalistas hacen remontarse la formación del superyó a una época más precoz", y ven actuar esta instancia desde las fases preedípicas (Melanie Klein), o por lo tríenos (sic.) buscan comportamientos y mecanismos psicológicos muy precoces que constituirían precursores del superyó (por ejemplo, Glover, Spitz).

Notas enciclopédicas:

El libro Das Ich und das Es apareció en la tercera semana de abril de 1923, si bien Freud ya venía pensando en él al menos desde julio del año anterior (Jones, 1957, pág. 10-11). El 26 de setiembre de 1922, en el 7º Congreso Psicoanalítico Internacional celebrado en Berlín (el último al que asistió), leyó un breve trabajo titulado «Etwas vom Unbewussten» (Consideraciones sobre lo inconsciente), que preanunciaba el contenido de la obra.

Toda esta información se deberá subir a la base de datos previamente confeccionada, campo por campo de acuerdo con la plantilla elaborada. 


\section{Análisis de la voz superyó}

Un análisis más detallado de la voz superyó pone de manifiesta algunas cuestiones que resultan interesantes de remarcar:

1. La prontitud con que penetró la voz desde el alemán en la literuatura especializada de la mano del la traducción de López-Ballesteros. En este caso se constata, además, que fue el español la segunda lengua de recepción del neologismo por detrás del francés y junto con el inglés.

2. La tardía incorporación del término en la lexicografía académica, muy por detrás del uso que se hace de ella.

3. Los ámbitos de uso. Con repecto a los ejemplos extraídos de la $H D$ podemos discernir los siguientes ámbitos en los que se refleja el término en la prensa periódica entre 1928 y 1933. El primero de ellos es una revista militar; aquí, no obstante, el uso que se hace de la voz no corresponde necesariamente con al significado acuñado por Freud; el autor lo utiliza como sinónimo de 'alma', según se comprueba en el texto, lejos del contenido propuesto por Freud. El segundo aparece en una noticia que explica el extraño suceso de 'El vampiro de Düsseldorf', con una explicación que se insiere en la esfera de la psiquiatría forense. El tercero, con el anuncio de una conferencia en el ámbito de la criminalogía y, por último, un comentario algo jocoso en el ámbito del deporte, concretamente del fútbol. Podría decirse que, con estos cuatro primeros ejemplos, el abanico de campos - y registros - en el que se ha utilizado el término abarca parcelas muy diversas de la sub-cultura social. Estos contextos resultan especialmente reveladores en lo que concierne a la penetración y uso del término en el cuarto ejemplo. Así, el último fragmento manifiesta un desplazamiento semántico notable para el momento, lo que nos lleva a pensar no solo en la pronta incorporación del término en la lengua especiaizada, sino el grado de aceptación de la voz en un uso que ya ha padecido el trasvase a la lengua común (Cabré 1993; Gutiérrez Rodilla 1998).

Concerniente a los contexto hallados en la $B V P H$ hemos escogido, asimismo, cuatro fragmentos, desde 1931 hasta 1940. El primero de ellos coincide con el de la HD. El segundo trata de las anomalías en la conducta infantil. Los dos siguientes se refieren a la psiquiatría forense. El primero es de 1935; el segundo, de 1940.

4. La ideología. El conjunto de los datos cognitivos manifiesta algunas singularidades de la relación entre léxico / sociedad, de las ideologías que transitan la época. Sin ir más lejos, el fragmento de la Hoja Oficial del lunes (1934) sugiere de forma desacomplejada que las anomalías en el comportamiento de los niños provienen de la 'torpeza de la madre'15. Con respecto al último ejemplo de la $B V P H$ la carga ideológica es más que evidente, lo que apoya la sugerencia hecha por Rubén (1993-1994).

15 De acuerdo con Laplanche-Pontalis, "Clásicamente el superyó se define como el heredero del complejo de Edipo; se forma por interiorización de las exigencias y prohibiciones parentales. [...] El superyó aparece principalmente como una instancia que encarna una ley y prohíbe su transgresión” (s.v.). Tratándose de 'prohibiciones parentales', no parece que la culpa haya de recaer exclusivamente en la madre. La mención al 'padre Moore' puede estar relacionada con el giro ideológico llevado a cabo por él y por el padre Gemelli, quienes manifiestan que el psicoanálisis debe ser interpretado con un sentido cristiano (Sánchez-Barranco Ruiz 2012). 


\section{A modo de conclusión}

Hasta quí solo hemos ejemplificado una única incidencia; la voz superyó. La plantilla de la base de datos que se ha diseñado, no obstante, irá incluyendo unidades terminológicas de acuerdo con el siguiente esquema simplificado en el cual, según se comprueba, se han seleccionado exclusivamente siete campos de los 33 previstos, a la par que se ha reducido la denominación de estos.

\begin{tabular}{|c|c|c|c|c|c|c|}
\hline 표 & छีّ & 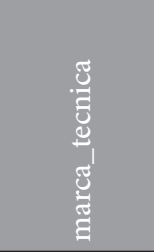 & 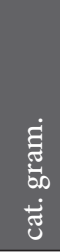 & 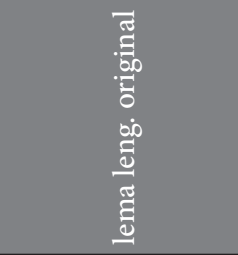 & 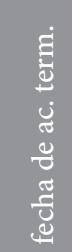 & 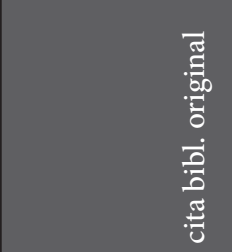 \\
\hline & superyó & psicoanálisis & s.m & Über-ich & 1923 & Das ich u. das Es \\
\hline & $\begin{array}{l}\text { complejo de } \\
\text { castración }\end{array}$ & psicoanálisis & s.m & Kastrationskomplex & 1923 & \begin{tabular}{|l|} 
Die infantile \\
Genitalorganisation
\end{tabular} \\
\hline & $\begin{array}{l}\text { complejo de } \\
\text { Edipo }\end{array}$ & psicoanálisis & s.m & Ödipuskomplex & 1913 & Totem u. Tabú \\
\hline
\end{tabular}

Esquema 3. Base de datos simplificada.

Los lemas se irán obteniendo de las obras de Freud. Inicialmente se vaciarán las tres obras del psicoanalista reflejadas en el esquema 2., esto es: Das ich und das Es, Die infantile Genitalorganisation, Totem und Tabú. A partir de aquí se irán añadiendo textos originales. El proyecto queda abierto a posibles ampliaciones, muy en especial, al número de lenguas analizado, primer registro en los diccionarios de otras lenguas, etc.

En cuanto a los resultados del proyecto cabe esperar que nos llevarán a conclusiones similares a las halladas para el término superyó. De manera más general, el alcance de aquel pretende ser una ayuda en el conocimiento de la evolución de la lengua española especializada. Por un lado, el cotejo con lo ocurrido en otras lenguas, el estudio de lo que contienen los diccionarios, así como la penetración de la terminología en los textos de la prensa periódica contribuirán a llenar una laguna importante en la descricpión de la lengua española. Por el otro, el establecimiento de un corpus de voces especializadas en el ámbito del psicoanálisis y su posterior plasmación en un producto lexicográfico digital facilitará el análisis lingüístico de los términos. Por último, los resultados de la propuesta esbozada también revestirán interés para los historiadores de la lingüística y, muy en especial, para los historiógrafos y para los historiadores de la ciencia y de la técnica, ya que podrán conocer mejor las vías de penetración de las ideas científicas en Europa y EEUU a través de la historia de las palabras ${ }^{16}$. El proyecto permitirá un estudio más amplio del uso de las voces tanto en la literatura especializada como en la no especializado a fin de poner de manifiesto las cuestiones hasta aquí detalladas.

16 Thomas Verner Moore (1877-1969) fue un psicólogo / psiquiatra monje. Desarrolló la psiquiatría basada en los fundamentos del catolicismo. De forma similar, tal y como se ha comentado en líneas anteriores, la aceptación del psicoanálisis en Italia no fue precisamente fácil. 


\section{Referencias bibliográficas}

\section{Bibliografía general}

Abaúnza, A. (1930). Prólogo a August Marie: La crisis del psicoanálisis. Madrid: Historia Nueva, vol. VII-XV. Amar, N.; Goues, G. Le; \& Pragier, G. (Eds.). (1995). Surmoi II: les developpements post-Freudiens. Paris: Presses Universitaires de France.

Azorín, D. (2011). Apuntes de la asignatura Terminología I: Lexicología y lexicografía aplicadas a la traducción. Tema: La terminología como disciplina: definición, funciones y aplicaciones. Grupo EPA. Alicane: Universidad de Alicante.

Brush, S. G. (1987). The History of modern Science: a Guide to the second scientific Revolution, 1800-1950. Ames: Iowa State University Press.

. (1991). Historia de la ciencia y enseñanza de las ciencias. Comunicación, lenguaje y educación, $11-12,169-180$.

Cabré, M. T. (1993). La terminología. Teoría, metodología y aplicaciones. Barcelona: Antárdida / Empúries.

Cabré, M.T.; \& Feliu, J. (2001). La terminología científico-técnica: Reconocimiento, análisis y extracción de información formal y semántica. Barcelona: IULA, Universitat Pompeu Fabra

Camacho, J. (2018). La Teoría funcional de la lexicografía y los diccionarios de especialidad lexicográfica. Propuesta microestructural. TFM, UNED.

Campos, M.; \& Pascual, J. A. (2012). Lexicografía, filología e informática: una alianza imprescindible. A propósito de la situación del NDHE. In D. Corbella et al. (Eds.), Lexicografía hispánico del siglo XXI, nuevos proyectos y perspectivas: homenaje al profesor Cristóbal Corrales Zumbado, (pp. 151-170). Madrid: Arco/ Libros.

Cotino, A. (1972). Ciencia y lenguaje. Discurso de recepción. RAE. Madrid: Talleres gráficos Vda. de C. Bermejo.

Donnet, J. L. (1995). Surmoi I: le concept Freudien et la regle fondamentale. Paris: P.U.F.

Fernández, J. et al. (eds.) (1982). I Simposium sobre metodología de historia de las ciencias. Llull, 5, 8-9, 209-250.

Fuertes-Olivera, P. A. (2012). La lexicografía de internet: el 'Diccionario inglés español de contabilidad'. Círculo de Lingüística de la Comunicación, (52), 21-56.

Fuertes-Olivera, P. A. (ed.) (2018). The Routledge Handbook of Lexicography. Londres/Nueva York: Routledge.

Fuertes-Olivera, P. A.; \& Tarp, S. (2014). Theory and Practice of Specialised Dictionaries. Lexicography versus Terminography). Berlín (Alemania), Boston: De Gruyter Mouton.

- (2008). La Teoría Funcional de la lexicografía y sus consecuencias para los diccionarios de economía del español. Revista de Lexicografía, XVI, 75-95.

Gago, R. (1982). Breve guía para la investigación en historia de la ciencia. Llull, 5, 8-9, 210-216.

Garriga, C. (2019). La lengua y el tecnicismo en el siglo XX. In M. Silva Suárez (Coord.), Técnica e ingeniería en España, 9, 109-170.

- (2009). La historia de la lengua científico-técnica y la traducción. Panace@: Revista de Medicina, Lenguaje y Traducción, 10, 30, 115-116.

Gómez de Enterría, J. (2003-2004). Estudio de los lenguajes especializados en español (I): elaboración y desarrollo de vocabularios científicos y técnicos. Linred, 1. <https://ebuah.uah.es/dspace/bitstream/handle/10017/24743/Estudio_Gomez_LR_2003_01.pdf?sequence=1\&isAllowed=y > 
Gouws, R. H.; \& Tarp, S. (2016). Information Overload and Data Overloading in Lexicography. International Journal of Lexicography, 30, 4, 389-415.

Gutiérrez Cuadrado, J. (2001). Lengua y ciencia en el siglo XIX español: el ejemplo de la química. In M. Bargalló et al. (Eds.), Las lenguas de especialidad y su didáctica (pp. 181-196). Tarragona: Universitat Rovira i Virgili.

Gutiérrez Rodilla, B. M. (1998). La ciencia empieza en la palabra. Análisis e historia del lenguaje científico. Barcelona: Península.

Mancho, Ma J. (2012). El Diccionario de la Ciencia y de la Técnica del Renacimiento (DICTER): un puente filológico entre las dos orillas del conocimiento. In D. Corbella et al. (eds.). Lexicografía hispánica del siglo XXI, nuevos proyectos y perspectivas: homenaje al profesor Cristóbal Corrales Zumbado (pp. 401-420). Madrid: Arco/Libros.

. (2014). La difusión del léxico científico y técnico español del Renacimiento en la red: el caso del Dicter. En I. Baraiber (Ed.), Visibilidad y divulgación de la investigación desde las humanidades digitales. Experiencias y proyectos (pp. 329-342). Navarra: Publicaciones digitales del GRISO.

Mandonlini, R. G. (1969). Historia general del psicoanálisis: de Freud a Fromm. Buenos Aries: Editorial Ciordia.

Martín, J.M.; \& Gallego, L. (2011). Luis López-Ballesteros: primer traductor de las obras completas de Freud al castellano.Panace@, 12, 34, 309-314.

Navarro, V. (1983). Historia de la ciencia y enseñanzas. In Actas del Simposio ICE (pp. 50-54). Valencia: Universidad de Valencia.

Pascual, J. A.; \& García Pérez, R. (2007). Límites y horizontes en un diccionario histórico. Salamanca: Difusión y publicaciones del departamento de Cultura-Diputación de Salamanca.

Peset, J. L. (1982). Problemas en historia de la ciencia. Llull, 5, 8, 217-219.

Rubén, J. (1993-1994). Las relaciones entre ciencia y sociedad: hacia una sociología histórica del conocimiento científico. Política y Social, 14, 15, 35-45.

Sánchez-Barranco Ruiz, A.; Sánchez-Barranco Vallejo, P.; \& Balbuena Rivera, F. (2012). Una contribución a la historia del psicoanálisis en España. Apuntes de Psicología, 30, 1-3, 165-174.

Tarp, S. (2000). Theoretical Challenges to Practical Specialised Lexicography. Lexikos, 10, 189-208.

. (2008). Lexicography in the Borderland between Knowledge and Non-Knowledge. General Lexicographical Theory with Particular Focus on Learner's Lexicography. Tübingen: Max Niemeyer Verlag.

. (2013a). Lexicographical functions. En Gouws, R. H. et al. (eds.). Dictionaries. An International Encyclopedia of Lexicography. Supplementary Volume: Recent Developments with Focus on Electronic and Computational Lexicography (pp. 460-468). Berlín, Boston: De Gruyter Mouton. . (2013b). Necesidad de una teoría independiente de la lexicografía: el complejo camino de la Lingüística teórica a la lexicografía práctica. Círculo de Lingüística Aplicada a la Comunicación, 56, 110-154. - (2015a). Presentación del Centro de lexicografía de Aarhus. Estudios de lexicografía, 4, 5-14. . (2015b). La teoría funcional en pocas palabras, en Estudios de lexicografía, 4, 31-42.

Vidal, M. (2013). El lenguaje del psicoanálisis. Temas de psicoanálisis, 6. <http://www.temasdepsicoanalisis. org/el-lenguaje-del-psicoanalisis-2/>

. (2014). El léxico del psicoanálisis en catalán: Emili Mira, la primera recepción de la terminología psicoanalítica. Temas de psicoanálisis, 7. <http://www.temasdepsicoanalisis.org/el-lexico-del-psicoanalisis -en-catalan-emili-mira-la-primera-recepcion-de-la-terminologia-psicoanalitica/> 


\section{Diccionarios de psicoanálisis}

Callicó, G. (1996). Vocabulari d psicoanàlisi. Barcelona: EUB, S.L.

Hinshelwood, R. D. (1989). Diccionario del pensamiento kleiniano. Trad. J. L. Etcheverry. Buenos Aires: Amorrortu editores.

Laplanche, J.; \& Pontalis, J. B. (1967). Diccionario de psicoanálisis. Barcelona: Paidós.

Roudinesco, E.; \& Plon, M. (1998). Diccionario de psicoanálisis. Barcelona: Paidós.

Sandler, P. C. (2009). A clinical application of Bion's concepts. 3 vols. London: Karnac Books.

VV.AA. (2002). International dictionary of psychoanalysis. Detroit, San Francisco, New York: The Thomson Corporation.

\section{Diccionarios de lengua}

[DLE]: Diccionario de la Lengua Española. <http://dle.rae.es>

[TLFi]: Trésor de la Langue Française informaticé. <http://atilf.atilf.fr/>

[OED]: Simpson J. A.; \& Weiner, E. S. C. (Eds.). Oxford English Dictionary. (1989). Oxford: Clarendon Press. [DELI]: Dizionario etimologico della lingua italiana. (1988). Bologna: Zanichelli.

Paul, H. (1846-1921/2002). Deutsches Wörterbuch: Bedeutungsgeschichte und Aufbau unseres Wortschatzes. Tübingen: Niemeyer.

Pfeifer, W.; \& Braun, W. (1993). Etymologisches Wörterbuch des Deutschen. Leipzig: Akademische Verlagsgesellschaft.

Grimm, J.; \& Grimm, W. (1838-1961). Deutsches Wörterbuch (DWB). <http://woerterbuchnetz.de/cgi-bin/ WBNetz/wbgui_py?sigle=DWB>

\section{Obras de referencia de Sigmund Freud}

Freud, S. (1913/1922). Totem und Tabu. Leipzig, Wien, Zürich: Internationaler psychoanalytischer Verlag.

- (1921). Massenpsychologie und Ich-Analyse. Frankfurt am Mein: S. Fischer Verlag.

- (1923). Das Ich und das Es. Frankfurt am Mein: S. Fischer Verlag.

- (1924). Zur Einführung des Narzissmus. Leipzig, Wien, Zürich: Internationaler psychoanalytischer Verlag

. (1930). Das Unbehagen in der Kultur. Wien: Internationaler psychoanalytischer Verlag.

. (1933). Neue Folge der Vorlesungen zur Einführung in die Psychoanalyse. Frankfurt am Mein: S.

Fischer Verlag. . (1939). Der Mann Moses und die monotheistische Religion. Frankfurt am Mein: S. Fischer Verlag. 


\section{Textos base del corpus}

Freud, S. (1922/34). Una teoría sexual y otros ensayos. Trad. L. López-Ballesteros. Madrid: Biblioteca Nueva. . (1923). Introducción a la psicoanálisis. Trad. L. López-Ballesteros. Madrid: Biblioteca Nueva.

. (1924/1934). La Psicología de las masas y Análisis del yo. Trad. L. López-Ballesteros. Madrid: Biblioteca Nueva.

- (1934). Nuevas aportaciones a la psicoanálisis y otros ensayos. Trad. L. López-Ballesteros. Madrid: Biblioteca Nueva.

\section{- (1934). Una teoría sexual y otros ensayos. Trad. L. López-Ballesteros, Madrid: Biblioteca Nueva.} . (1931-1934). La interpretación de los sueños. 2 vols. Trad. L. López-Ballesteros. Madrid: Biblioteca Nueva.

(1976). Obras completas. Trad. J. L. Etxeverry. Buenos Aires: Ediciones Amorrutu.

\section{Páginas web}

Dicter $<$ http://dicter.usal.es/>

Freud, S. <http://www.psyalpha.net/literatur/literaturverzeichnisse/freud-sigmund-gesammelte-werke-inhaltsverzeichnis>

Freud, S. <http://www.psychotherapie-graz.info/freud-psychoanalyse-graz/Freud_GW_I.pdf.>

Hemeroteca Digital de la BNE <http://www.bne.es/ca/Catalogos/HemerotecaDigital/>

Biblioteca Virtual de Prensa Histórica <https://prensahistorica.mcu.es/es/inicio/inicio.do>

Lexilogos $<$ https://www.lexilogos.com/ $>$

NDHE <http://www.rae.es/recursos/diccionarios/nuevo-diccionario-historico >

Project Gutenberg <https://www.gutenberg.org/catalog/>

SEP (Sociedad española de psicoanálisis). <http://www.sep-psicoanalisi.org/ca/publicacions/temas-de-psicoanalisis/> 
